Georgian Mathematical Journal

Volume 8 (2001), Number 4, 697-712

\title{
SUSPENSION AND LOOP OBJECTS IN THEORIES AND COHOMOLOGY
}

\author{
H.-J. BAUES AND M. JIBLADZE
}

\begin{abstract}
We introduce theories in which suspension, resp. loop objects, are defined and we describe the cohomology of such theories. Cohomology classes in degree 3 classify track theories.
\end{abstract}

2000 Mathematics Subject Classification: 18D05, 55P35, 55P40.

Key words and phrases: Theory with suspension objects, theory with loop objects, $\Sigma$-cohomology, $\Omega$-cohomology, track theory.

Since the work of Lawvere [16] it is well known that many algebraic categories (like the categories of monoids, groups, algebras, Lie algebras, etc.) are actually categories of models of a theory. In topology there are fundamental examples of theories as in (1) and (2) below in which also suspension objects $\Sigma X$ or loop objects $\Omega X$ are defined. Such theories with suspension objects are also endowed with a partial suspension operator $E$ and dually theories with loop objects are endowed with a partial loop operator $L$, compare for example Baues [5, 4, 2].

For example the homotopy category $\mathbf{S}$ of one point unions of spheres

$$
S^{n_{1}} \vee \ldots \vee S^{n_{k}}
$$

with $n_{1}, \ldots, n_{k} \geq 1, k \geq 0$, is a theory with suspension objects and partial suspension operator $E$. Moreover the homotopy category $\mathbf{K}(\mathbb{Z} / 2)$ of products of Eilenberg-Mac Lane spaces

$$
K^{n_{1}} \times \ldots \times K^{n_{k}}
$$

with $n_{1}, \ldots, n_{k} \geq 0, k \geq 0$ and $K^{n}=K(\mathbb{Z} / 2, n)$ is a theory with loop objects and partial loop operator $L$.

In this paper we introduce a complete system of axioms defining a theory with suspension objects and partial suspension operator termed a $\Sigma$-theory. The categorical dual of a $\Sigma$-theory is a theory with loop objects and partial loop operator termed $\Omega$-theory.

The theory $\mathbf{S}$ given by spheres in (1) is a $\Sigma$-theory and the theory $\mathbf{K}(\mathbb{Z} / 2)$ given by Eilenberg-Mac Lane spaces in (2) is an $\Omega$-theory. These examples show the crucial significance of such theories. In [10] we show that the $\Omega$-theory $\mathbf{K}(\mathbb{Z} / 2)$ is completely determined by the Steenrod algebra.

We introduce the $\Sigma$-cohomology $H_{\Sigma}^{*}(\mathbf{T})$ of a $\Sigma$-theory $\mathbf{T}$ and dually the $\Omega$ cohomology $H_{\Omega}^{*}(\mathbf{T})$ of an $\Omega$-theory $\mathbf{T}$. The universal Toda brackets of Baues and Dreckmann [6] yield for the theories $\mathbf{S}$ and $\mathbf{K}(\mathbb{Z} / 2)$ above the cohomology 
classes

$$
\begin{array}{r}
\langle\mathbf{S}\rangle \in H_{\Sigma}^{3}(\mathbf{S}), \\
\langle\mathbf{K}(\mathbb{Z} / 2)\rangle \in H_{\Omega}^{3}(\mathbf{K}(\mathbb{Z} / 2)) .
\end{array}
$$

The cohomology class $\langle\mathbf{S}\rangle$ determines all secondary homotopy operations in $\mathbf{S}$ like triple Toda brackets. The cohomology class $\langle\mathbf{K}(\mathbb{Z} / 2)\rangle$ determines all secondary cohomology operations like triple Massey products or operations of Adams [1].

In [9] we study the concept of suspenders and loopers in groupoid enriched categories termed track categories. As a main result in this paper we show that a $\Sigma$-theory $\mathbf{T}$ together with an element $\alpha \in H_{\Sigma}^{3}(\mathbf{T})$ determines up to equivalence a track category which has the property of a "track theory with suspenders" and vice versa such track theories are classified by the $\Sigma$-cohomology $H_{\Sigma}^{3}$.

Dually an $\Omega$-theory $\mathbf{T}$ together with an element $\alpha \in H_{\Omega}^{3}(\mathbf{T})$ determines up to equivalence a track category which has the properties of a "track theory with loopers" and vice versa such track theories are classified by the $\Omega$-cohomology $H_{\Omega}^{3}$.

For example the topological track category given by spaces in (1) and maps between such spaces (1-cells) and by homotopy classes of homotopies between such maps (2-cells) is a track theory with suspenders corresponding to the pair $(\mathbf{S},\langle\mathbf{S}\rangle)$. Similarly the topological track category given by spaces in (2) is a track theory with loopers corresponding to the pair $(\mathbf{K}(\mathbb{Z} / 2),\langle\mathbf{K}(\mathbb{Z} / 2)\rangle)$.

\section{THEORIES}

A theory for us is a category $\mathbf{S}$ in which finite sums exist. We assume that a collection of generating objects $X_{i}$ in $\mathbf{S}$ is given such that all objects of $\mathbf{S}$ are finite sums of objects from the collection. A model $M$ of the theory $\mathbf{S}$ in a category $\mathbf{C}$ is a contravariant functor $M: \mathbf{S}^{\mathrm{op}} \rightarrow \mathbf{C}$ which carries sums to products. Hence a model can be thought of as a collection of objects $M_{i}$ of $\mathbf{C}$ together with certain morphisms ("operations") $M_{i_{1}} \times \ldots \times M_{i_{n}} \rightarrow M_{i}$ which satisfy certain "identities", i. e. fit in certain commutative diagrams, according to the structure of $\mathbf{S}$. In most cases we consider models of $\mathbf{S}$ in the category Set of sets, so that a model is a collection of sets with algebraic structure.

1.1. Example. Let $\mathbf{S}$ be the full subcategory of the homotopy category $\left(\mathbf{T o p}^{*}\right)_{\simeq}$ consisting of finite one point unions of spheres $S^{n}$ with $n \geq 1$. Then $\mathbf{S}$ is a theory. Homotopy groups $\pi_{n}(X)=\left[S^{n}, X\right]$ of a space $X$ together with homotopy operations given by maps in $\mathbf{S}$ yield a model of $\mathbf{S}$. Such models are also termed $\pi$-algebras in the work of Blanc [12], Stower [18], Dwyer-Kan-Stower [13], Baues-Goerss [7].

The categorical dual of a theory termed a theory with products is a category $\mathbf{P}$ with finite products denoted $A \times B$. A model of $\mathbf{P}$ is a covariant functor $\mathbf{P} \rightarrow \mathbf{C}$ which preserves products. Originally Lawvere used such theories with products. But the example of homotopy groups above shows that also theories with coproducts arise naturally. 
1.2. Example. Let $\mathbf{K}(\mathbb{Z} / 2)$ be the full subcategory of the homotopy category $\left(\text { Top }^{*}\right)_{\simeq}$ consisting of finite products of Eilenberg-MacLane spaces $K(\mathbb{Z} / 2, n)$ with $n \geq 1$. Then $\mathbf{K}$ is a theory with products. The cohomology groups $H^{n}(X ; A)=[X, K(A, n)]$ of a space $X$ together with cohomology operations given by maps in $\mathbf{K}(\mathbb{Z} / 2)$ yield a model of $\mathbf{K}(\mathbb{Z} / 2)$. For example, the cohomology of $X$ as a module over the Steenrod algebra or the cohomology ring of $X$ can be deduced from the model; see Gray [14].

Each object in $\mathbf{S}$ is a cogroup object and objects in $\mathbf{K}$ are group objects. Therefore $\mathbf{S}$ is a "theory of cogroups" as defined below and dually $\mathbf{K}$ is a "theory of groups".

1.3. Definition. A theory of cogroups is a theory $\mathbf{T}$ all of whose objects $S$ are equipped with a cogroup structure $\left(0_{S}: S \rightarrow *, m_{S}: S \rightarrow S \vee S, n_{S}: S \rightarrow S\right)$ in a way which is compatible with sums, i. e.

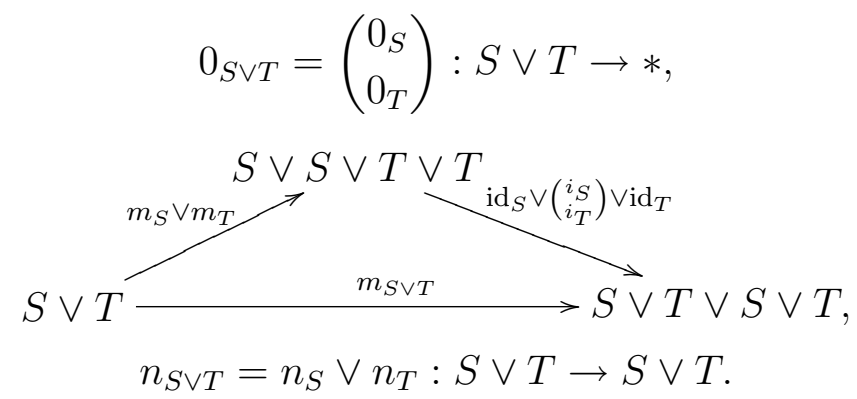

We shall deal more generally with the following theories of coactions which are fundamental in development of homotopy theory in [3]. Principal coactions are defined as in $[9$, Section 4$]$.

1.4. Definition. A theory of coactions is a theory $\mathbf{T}$ all of whose objects $X$ are assigned a cogroup $S_{X}$ and a principal coaction with structure maps $a_{X}: X \rightarrow$ $X \vee S_{X}, d_{X}: S_{X} \rightarrow X \vee X$. These structures are required to be compatible with sums, i. e. $S_{X \vee Y}=S_{X} \vee S_{Y}$, etc.

Clearly a theory of cogroups is a special case of a theory of coactions, with $S_{X}=X, a_{X}=m_{X}, d_{X}=\left(n_{X} \vee \operatorname{id}_{X}\right) m_{X}$, where $m_{X}$ and $n_{X}$ are the comultiplication and the coinverse respectively of the cogroup $X$.

1.5. Definition. Let $S$ and $T$ be cogroups in a category C. We say that $f: S \rightarrow T$ is linear if $f$ is compatible with the cogroup structure, i. e. $m_{T} f=$ $(f \vee f) m_{S}$. More generally a map $f: S \rightarrow X \vee T$ is called linear in $T$ if the diagram

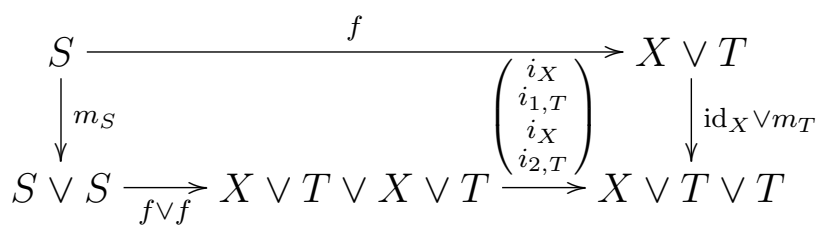


commutes.

We point out that in a theory of cogroups $\mathbf{T}$, morphisms in $\mathbf{T}$ are not required to be linear.

\section{TheORIES WITH SUSPENSION}

In this section we introduce and study "theories with suspension objects and partial suspension operator" termed $\Sigma$-theories. There are three different ways to characterize a $\Sigma$-theory. The categorical dual of a $\Sigma$-theory is termed a $\Omega$-theory.

Let $\mathbf{T}$ be a theory of cogroups or more generally a theory of coactions.

For an object $X$ of $\mathbf{T}$ we define the category $\mathbf{T}(X)$ as follows. Objects of $\mathbf{T}(X)$ are the objects of $\mathbf{T}$ having a cogroup structure. A morphism $s$ from $S$ to $T$ in $\mathbf{T}(X)$ is given by a commutative diagram

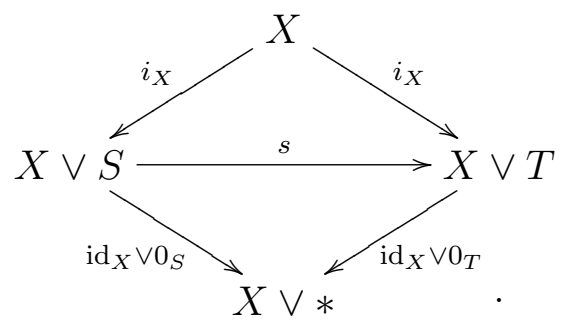

Note that each map $f: X \rightarrow Y$ induces a functor $\mathbf{T}(f): \mathbf{T}(X) \rightarrow \mathbf{T}(Y)$ which is identity on objects and carries $a: X \vee S \rightarrow X \vee T$ to

$$
\left(\begin{array}{c}
i_{Y} \\
(f \vee T) a i_{S}
\end{array}\right): Y \vee S \rightarrow Y \vee T
$$

In particular $\mathbf{T}(*)$ is the category whose objects are cogroups in $\mathbf{T}$ and whose morphisms are maps respecting counits. It is thus a theory of cogroups, and it is easy to see that the functor $\mathbf{T}\left(!_{X}\right): \mathbf{T}(*) \rightarrow \mathbf{T}(X)$ induced as above preserves finite coproducts. Hence also $\mathbf{T}(X)$ is a theory of cogroups with the same structure on objects as $\mathbf{T}(*)$.

There is an alternative description of morphisms in $\mathbf{T}(X)$ as follows: assigning to $s: X \vee S \rightarrow X \vee T$ the map $s i_{S}: S \rightarrow X \vee T$ gives an exact sequence of groups

$$
0 \rightarrow \operatorname{hom}_{\mathbf{T}(X)}(S, T) \longrightarrow \operatorname{hom}_{\mathbf{T}}(S, X \vee T) \stackrel{\operatorname{hom}_{\mathbf{T}}\left(S, \operatorname{id}_{X} \vee 0_{T}\right)}{\longrightarrow} \operatorname{hom}_{\mathbf{T}}(S, X \vee *),
$$

where the group structures are induced by the cogroup structure of $S$. In other words, there are isomorphisms

$$
\operatorname{hom}_{\mathbf{T}(X)}(S, T) \cong[S, X \vee T]_{X}
$$

where by definition $[S, X \vee T]_{X}=\operatorname{hom}_{\text {Pair(T) }}\left(0_{S}, \operatorname{id}_{X} \vee 0_{T}\right)$. This is the set of those maps $S \rightarrow X \vee T$ which are "trivial on $X$ ", i. e. those maps $g: S \rightarrow X \vee T$ 
which render the diagram

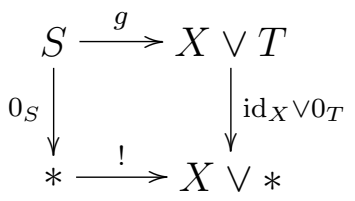

commutative. The inverse isomorphism $[S, X \vee T]_{X} \cong \operatorname{hom}_{\mathbf{T}(X)}(S, T)$ carries such a map $g$ to

$$
\left(\begin{array}{c}
i_{X} \\
g
\end{array}\right): X \vee S \rightarrow X \vee T
$$

It is easy to see that $s: X \vee S \rightarrow X \vee T$ is linear as a morphism of $\mathbf{T}(X)$ iff $s i_{S}$ : $S \rightarrow X \vee T$ is linear in $T$ as a morphism in $\mathbf{T}$; see 1.5. The following definition of a $\Sigma$-theory is canonically derived from the notion of a $\Sigma$-representable track category as we shall see in our main result 4.2 below.

2.1. Definition. A theory with suspensions (or a $\Sigma$-theory for short) is a theory of coactions $\mathbf{T}$ together with a system of functors

$$
E_{X}: \mathbf{T}(X) \rightarrow \mathbf{T}(X)
$$

which preserve finite sums and carry each map to a linear map. These functors must be natural in the sense that for all maps $f: X \rightarrow Y$ the diagram of functors

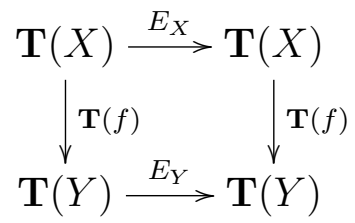

commutes. In particular for $X=*$ one has the suspension functor

$$
\Sigma=E_{*}: \mathbf{T}(*) \rightarrow \mathbf{T}(*) .
$$

Hence for each cogroup $S$ in $\mathbf{T}$ the suspension $\Sigma S$ is defined and is a cogroup in T. Considering the above diagram for $f=!_{X}: * \rightarrow X$ one sees that all functors $E_{X}$ coincide with $\Sigma$ on objects. Moreover using the identification (2.2) above one may describe the effect of $E_{X}$ on morphisms by maps which we will call partial suspension maps

$$
E:[S, X \vee T]_{X} \rightarrow[\Sigma S, X \vee \Sigma T]_{X}
$$

so that

$$
E_{X}\left(\begin{array}{c}
i_{X} \\
g
\end{array}\right)=\left(\begin{array}{c}
i_{X} \\
E g
\end{array}\right) .
$$

By definition, for any map $f: S \rightarrow T$ in $\mathbf{T}(*)$, the map $\Sigma f$ is linear. More generally for any $g: S \rightarrow X \vee T$ with $\left(\operatorname{id}_{X} \vee 0_{T}\right) g=i_{*} 0_{S}: S \rightarrow X \vee *$, the map $E g: \Sigma S \rightarrow X \vee \Sigma T$ is linear in $\Sigma T$. Considering the comultiplication $m_{S}: S \rightarrow S \vee S$ as a morphism in $\mathbf{T}(*)$ one sees that

$$
\Sigma m_{S}: \Sigma S \rightarrow \Sigma(S \vee S)=\Sigma S \vee \Sigma S
$$

is linear. This implies that the cogroup structure on $\Sigma S$ is coabelian. 
2.2. Remark. The categorical dual of a theory with suspensions ( $\Sigma$-theory) is a theory with loop objects termed an $\Omega$-theory for short. Hence an $\Omega$-theory $\mathbf{T}$ is a category with finite products in which each object $X$ is endowed with an action of a group object $G, X \times G \rightarrow X$, so that $\mathbf{T}$ is a theory of actions with properties dual to 1.4. Moreover $\mathbf{T}(X)$ is the category of diagrams

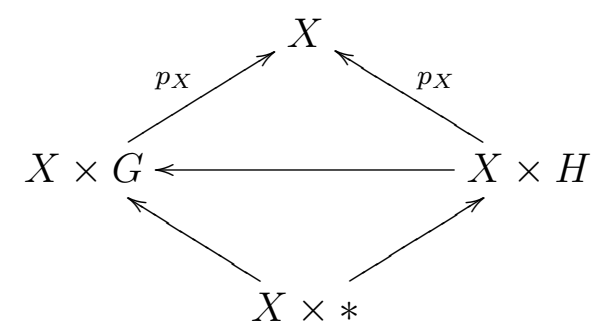

dual to (2.1) and a system of functors

$$
L_{X}: \mathbf{T}(X) \rightarrow \mathbf{T}(X)
$$

is given with properties dual to 2.1. The loop functor is given by

$$
\Omega=L_{*}: \mathbf{T}(*) \rightarrow \mathbf{T}(*)
$$

which carries a group object $G$ to the group object $\Omega G$ in T. Moreover partial loop operations

$$
L:[X \times H, G]_{X} \rightarrow[X \times \Omega H, \Omega G]_{X}
$$

are defined dually to $(2.3)$ by $L_{X}$ in (2) above.

There are alternative ways to characterize a $\Sigma$-theory:

2.3. Lemma. Equipping a theory of coactions $\mathbf{T}$ with the structure of a $\Sigma$ theory is equivalent to assigning a cogroup $\Sigma S$ to each cogroup $S$ in $\mathbf{T}$ and functions

$$
E:[S, X \vee T]_{X} \rightarrow[\Sigma S, X \vee \Sigma T]_{X}
$$

to each object $X$ and cogroups $S, T$ in such a way that the following conditions are satisfied.

$$
E\left(\left(\begin{array}{l}
f \\
b
\end{array}\right) a\right)=\left(\begin{array}{c}
f \\
E b
\end{array}\right) E a
$$

for any $f: X \rightarrow Y, a \in[S, X \vee T]_{X}, b \in[T, Y \vee U]_{Y}$, and furthermore Ea is linear in $\Sigma T$. The functor $\Sigma$ obtained from the particular case of this condition when $X=Y=*$, must preserve sums. And, for $i_{S}: S \rightarrow X \vee S$ one must have $E i_{S}=i_{\Sigma S}: \Sigma S \rightarrow X \vee \Sigma S$.

Proof. We already saw how to derive partial suspension maps from the $\Sigma$-structure and the maps $E_{X}$ from $E$. Conditions that they must obey are equivalent, to properties of $E_{X}$, namely, to preservation of composition, sums and identities, respectively.

Conversely, given the maps $E$, one defines $E_{X}$ as above and then the required properties will correspond to properties of $E$ as indicated. 
For any $\Sigma$-theory $\mathbf{T}$ one has the functor

$$
\Sigma_{*}: \mathbf{T} \rightarrow \mathbf{T},
$$

termed the suspender of $\mathbf{T}$, as follows. For an object $X$ of $\mathbf{T}$ with the coaction structure $\left(S_{X}, a_{X}, d_{X}\right)$ let $\Sigma_{*}(X)=X \vee \Sigma S_{X}$. Moreover define the difference $\operatorname{map} \nabla(f) \in\left[S_{X}, Y \vee S_{Y}\right]_{Y}$ for any map $f: X \rightarrow Y$ by

$$
\nabla(f)=\left(S_{X} \stackrel{d_{X}}{\longrightarrow} X \vee X \stackrel{f \vee f}{\longrightarrow} Y \vee Y \stackrel{\left(\begin{array}{c}
i_{Y} \\
a_{Y}
\end{array}\right)}{\longrightarrow} Y \vee S_{Y}\right)
$$

Then the partial suspension in $\mathbf{T}$ yields $E \nabla(f) \in\left[\Sigma S_{X}, Y \vee \Sigma S_{Y}\right]$ and one defines

$$
\Sigma_{*}(f)=\left(\begin{array}{c}
i_{Y} f \\
E \nabla(f)
\end{array}\right): X \vee \Sigma S_{X} \rightarrow Y \vee \Sigma S_{Y}
$$

This indeed defines a functor $\Sigma_{*}$ which also can be used to characterize the $\Sigma$-theory completely.

2.4. Lemma. Let $\mathbf{T}$ be a theory of coactions. Then structures of a $\Sigma$-theory on $\mathbf{T}$ are in a one-to-one correspondence with sum-preserving endofunctors

$$
\Sigma_{*}: \mathbf{T} \rightarrow \mathbf{T}
$$

equipped, for each coaction $\left(X, S_{X}, a_{X}, d_{X}\right)$ in $\mathbf{T}$, with isomorphisms

$$
\vartheta_{X}: \Sigma_{*}(X) \cong X \vee \Sigma S_{X}
$$

for some cogroups $\Sigma S_{X}$ depending only on the cogroups $S_{X}$. These isomorphisms must be natural in the sense that the inclusions

$$
j_{X}=\vartheta_{X}^{-1} i_{X}: X \rightarrow \Sigma_{*}(X)
$$

and the projections

$$
p_{X}=\left(\begin{array}{c}
\operatorname{id}_{X} \\
0_{\Sigma S_{X}}
\end{array}\right) \vartheta_{X}: \Sigma_{*}(X) \rightarrow X
$$

are natural in $X$. Moreover the composite maps

$$
\Sigma S_{X} \stackrel{i_{\Sigma S_{X}}}{\longrightarrow} X \vee \Sigma S_{X} \stackrel{\vartheta_{X}^{-1}}{\longrightarrow} \Sigma_{*}(X) \stackrel{\Sigma_{*}(f)}{\longrightarrow} \Sigma_{*}(Y) \stackrel{\vartheta_{Y}}{\longrightarrow} Y \vee \Sigma S_{Y}
$$

must be linear in $\Sigma S_{Y}$.

Proof. Given the functor $\Sigma_{*}$ as above, for any cogroup $S$ consider $S$ as a coaction via $S_{S}=S$ coacting on itself via its own comultiplication; let $\Sigma S=\Sigma S_{S}$. These come equipped with maps $q_{S}: \Sigma_{*}(S) \rightarrow \Sigma S$ given by

$$
q_{S}=\left(\Sigma_{*}(S) \stackrel{\vartheta_{S}}{\longrightarrow} S \vee \Sigma S \stackrel{0_{S} \vee \mathrm{id}_{\Sigma_{S}}}{\longrightarrow} * \vee \Sigma S \cong \Sigma S\right)
$$


We call $\Sigma$ the suspension and define the partial suspension $E g$ for $g \in[S, X \vee T]_{X}$ by

$$
\begin{aligned}
E g=\left(\Sigma S \stackrel{i_{\Sigma S}}{\longrightarrow} S \vee \Sigma S \stackrel{\vartheta_{S}^{-1}}{\longrightarrow} \Sigma_{*}(S)\right. & \stackrel{\Sigma_{*}(g)}{\longrightarrow} \Sigma_{*}(X \vee T) \\
& \left.\cong \Sigma_{*}(X) \vee \Sigma_{*}(T) \stackrel{p_{X} \vee q_{T}}{\longrightarrow} X \vee \Sigma T\right)
\end{aligned}
$$

It is clear that $E g$ is linear in $\Sigma T$ and also other properties required of $E$ and $\Sigma$ are all satisfied. The converse procedure has been already described above.

\section{Cohomology of $\Sigma$-THeOries AND $\Omega$-THeOries}

Recall that a natural system $D$ on a category $\mathbf{T}$ is a functor $D: F \mathbf{T} \rightarrow$ Ab where $F \mathbf{T}$ is the category of factorizations of $\mathbf{T}$; see $[8,2.1]$. Here $D$ carries the object $f: A \rightarrow B$ in $F \mathbf{T}$ to an abelian group $D_{f}$ and carries maps $\leftarrow_{g}<\frac{}{f}<\frac{}{h}$ to induced action maps $g_{*}: D_{f} \rightarrow D_{g f}, \xi \mapsto g \xi$, and $h^{*}: D_{f} \rightarrow D_{f h}, \xi \mapsto \xi h$.

For objects $A, B$ in $\mathbf{T}$ let

$$
[A, B]=\operatorname{hom}(A, B)
$$

be the set of morphisms $A \rightarrow B$ in $\mathbf{T}$. If $\mathbf{T}$ is a $\Sigma$-theory we have for the coaction on each object $X$ in $\mathbf{T}$ the structure maps

$$
\left\{\begin{array}{l}
a_{X}: X \rightarrow X \vee S_{X} \\
d_{X}: S_{X} \rightarrow X \vee X
\end{array}\right.
$$

as in 1.4 .

3.1. Proposition. Each $\Sigma$-theory $\mathbf{T}$ comes equipped with a canonical natural system $D^{\mathbf{T}}$ defined as follows: For a map $f: X \rightarrow Y$ let

$$
D_{f}^{\mathbf{T}}=\left[\Sigma S_{X}, Y\right]
$$

with the abelian group structure induced by the abelian cogroup $\Sigma S_{X}$. Left action of a map $y: Y \rightarrow Y^{\prime}$ on $a: \Sigma S_{X} \rightarrow Y$ is given by

$$
y_{*}(a)=y a \text {. }
$$

The right action of $x: X^{\prime} \rightarrow X$ on a is given by the composite

$$
\Sigma S_{X^{\prime}} \stackrel{E \nabla(f)}{\longrightarrow} X \vee \Sigma S_{X} \stackrel{\left(\begin{array}{l}
f \\
a
\end{array}\right)}{\longrightarrow} Y
$$

where $\nabla(f)$ is the composite

$$
\nabla(f)=\left(S_{X} \stackrel{d_{X}}{\longrightarrow} X \vee X \stackrel{f \vee f}{\longrightarrow} Y \vee Y \stackrel{\left(\begin{array}{c}
i_{Y} \\
a_{Y}
\end{array}\right)}{\longrightarrow} Y \vee S_{Y}\right)
$$


Proof. It turns out that naturality of actions is evident if one uses the functor $\Sigma_{*}$ in (2.4) above, whereas the fact that this is indeed the natural system of abelian groups and not just sets is clear if one uses the suspensions $\Sigma$. So let us first define the actions using the former and then transform them using the latter.

For any morphism $f: X \rightarrow Y$, put

$$
D_{f}^{\mathbf{T}}=\left\{a: \Sigma_{*}(X) \rightarrow Y \mid a j_{X}=f\right\},
$$

as in the diagram

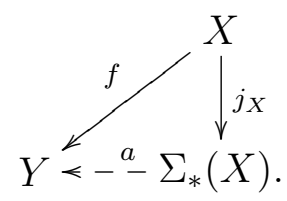

Actions of this natural system are defined as follows: for $y: Y \rightarrow Y^{\prime}$ let the induced action $D_{f}^{\mathbf{T}} \rightarrow D_{y f}^{\mathbf{T}}$ be given by $a \mapsto y a$, as in the diagram

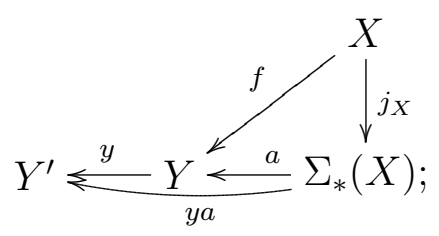

whereas for $x: X^{\prime} \rightarrow X$ let the action $D_{f}^{\mathbf{T}} \rightarrow D_{f x}^{\mathbf{T}}$ be given by $a \mapsto a \Sigma_{*}(x)$, as in the diagram

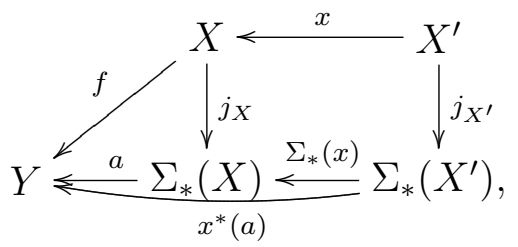

where the square commutes by 2.4 .

Now let us use the isomorphisms

$$
\vartheta_{X}: \Sigma_{*}(X) \cong X \vee \Sigma S_{X}
$$

inducing bijections

$$
D_{f}^{\mathbf{T}} \approx\left[\Sigma S_{X}, Y\right]
$$

where the latter sets have the group structure induced by the canonical cogroup structure on $\Sigma S_{X}$. In fact as we have seen $\Sigma S_{X}$ is an abelian cogroup (see 2.1), so the groups $D_{f}^{\mathbf{T}}$ are indeed abelian. Moreover the linearity conditions imply that the actions are indeed homomorphisms.

For convenience let us give explicit expressions for the actions in these new terms. Left actions are again given by composition: for $y: Y \rightarrow Y^{\prime}$ the induced homomorphism is

$$
\left[\Sigma S_{X}, Y\right] \rightarrow\left[\Sigma S_{X}, Y^{\prime}\right], \text { given by } a \mapsto y a
$$

for any $a: \Sigma S_{X} \rightarrow Y$. 
Right actions are more involved. For $x: X^{\prime} \rightarrow X$ and $a: \Sigma S_{X} \rightarrow Y$ the map $x^{*}(a): \Sigma S_{X^{\prime}} \rightarrow Y$ is given by the lower horizontal composition in the diagram

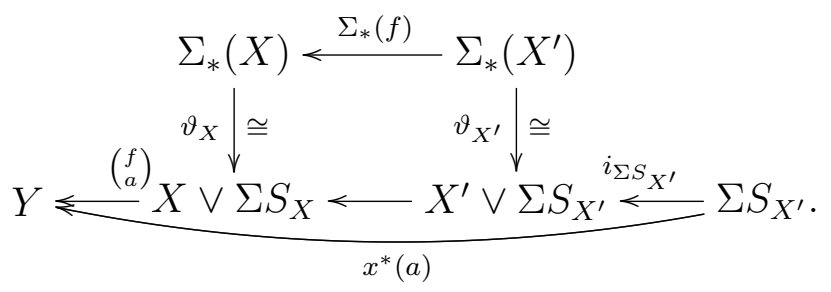

This yields the result by use of the definition of $\Sigma_{*}$ in $(2.4)$.

For each category $\mathbf{C}$ with natural system $D$ the cohomology $H^{n}(\mathbf{C} ; D)$ is defined as in [11]. This leads to the following notion of $\Sigma$-cohomology.

3.2. Definition. Let $\mathbf{T}$ be a $\Sigma$-theory and let $D^{\mathbf{T}}$ be the natural system determined by $\mathbf{T}$ as in 3.1. Then the $\Sigma$-cohomology of $\mathbf{T}$ is given by the abelian group $(n \geq 0)$

$$
H_{\Sigma}^{n}(\mathbf{T})=H^{n}\left(\mathbf{T} ; D^{\mathbf{T}}\right) .
$$

The categorical dual of a $\Sigma$-theory is an $\Omega$-theory $\mathbf{T}$ for which we have accordingly the $\Omega$-cohomology

$$
H_{\Omega}^{n}(\mathbf{T})=H^{n}\left(\mathbf{T} ; D^{\mathbf{T}}\right),
$$

where $D^{\mathbf{T}}$ is the natural system associated to $\mathbf{T}$ with $D_{f}^{\mathbf{T}}=[A, \Omega B]$ for $f: A \rightarrow$ $B$ in $\mathbf{T}$.

\section{TRACK THEORIES WITH SUSPENSIONS.}

A track theory $\mathscr{T}$ is a track category with sums $A \vee B$ in the weak sense so that for hom-groupoids in $\mathscr{T}$ the map

$$
\llbracket A \vee B, X \rrbracket \stackrel{\sim}{\longrightarrow} \llbracket A, X \rrbracket \times \llbracket B, X \rrbracket
$$

is an equivalence of groupoids; see $[8, \S 4]$. Moreover $\mathscr{T}$ is $\Sigma$-representable if suspenders $\left(\Sigma_{f}, i_{f}, v_{f}\right)$ as defined in [9] exist for all maps $f: A \rightarrow B$ in $\mathscr{T}$.

4.1. Definition. A $\Sigma$-track theory is a track theory $\mathscr{T}$ which is $\Sigma$-representable and for which the homotopy category $\mathscr{T} \simeq$ is a theory of coactions.

Recall that linear track extensions are defined as in $[8,2.2]$. It is clear that a $\sum$-track theory $\mathscr{T}$ has abelian hom-groupoids so that $\mathscr{T}$ is a linear track extension of its homotopy category $\mathscr{T} \simeq$ as in [8].

4.2. Theorem. For any $\Sigma$-track theory $\mathscr{T}$ its homotopy category carries a canonical structure of a $\Sigma$-theory and $\mathscr{T}$ is a linear track extension of $\mathscr{T} \simeq$ by

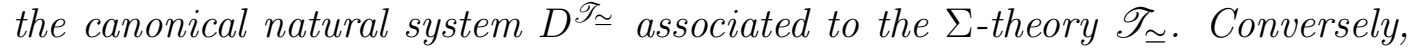
any linear track extension of a $\Sigma$-theory by its canonical natural system is a s-track theory. 
Proof. Consider a track category $\mathscr{T}$ satisfying the hypothesis. To equip $\mathscr{T} \simeq$ with a structure of a $\Sigma$-theory it suffices, according to 2.1, to define the functor $\Sigma_{*}$. We do this by declaring $\Sigma_{*}(X)=\Sigma_{\mathrm{id}_{X}}$ for all objects $X$ and $\Sigma_{*}(f)=\Sigma_{*}(f, f)$ for all maps $f: X \rightarrow Y$, where $(f, f)$ is considered as a morphism in $\operatorname{Pair}(\mathscr{T})$ from $\operatorname{id}_{X}$ to $\operatorname{id}_{Y}$. By $[9,2.1]$ this indeed defines an endofunctor, equipped with natural maps $j_{X}=i_{\operatorname{id}_{X}}: X \rightarrow \Sigma_{*}(X) . \Sigma_{*}$ preserves sums by [9, 2.4]. Moreover the isomorphisms $\vartheta$ are obtained, in view of $[9,2.2]$, by virtue of $[9,4.3]$. The latter also implies that under these isomorphisms the maps $j_{X}$ correspond to the coproduct inclusions $i_{X}: X \rightarrow X \vee \Sigma S_{X}$. To prove naturality of the projections

$$
p_{X}=\left(\begin{array}{c}
\operatorname{id}_{X} \\
0_{\Sigma S_{X}}
\end{array}\right) \vartheta_{X}: \Sigma_{*}(X) \rightarrow X
$$

it suffices to construct, for any map $f: X \rightarrow Y$, a track $f p_{X} \simeq p_{Y} \Sigma_{*}(f)$. By the universal property of the suspender $\Sigma_{\mathrm{id}_{X}}$, such track exists iff there is a track in $\mathbf{G}_{\mathrm{id}_{X}}$ between $f p_{X} v_{X} \in \operatorname{Aut}\left(f p_{X} j_{X}\right)$ and $p_{Y} \Sigma_{*}(f) v_{X} \in \operatorname{Aut}\left(p_{Y} \Sigma_{*}(f) j_{X}\right)$. By $[9,2.1]$, one may take $\zeta_{(f, f)}$ for this purpose. Finally, linearity condition from 2.1 means commutativity in $\mathscr{T}$ of the diagram

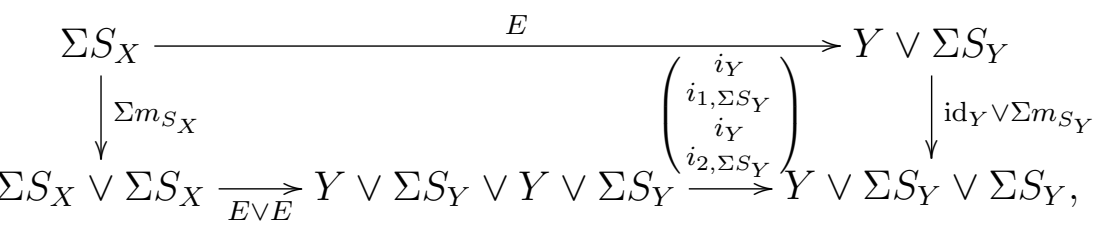

for $f: X \rightarrow Y$, where $E$ is the composite

$$
\Sigma S_{X} \stackrel{i_{\Sigma S_{X}}}{\longrightarrow} X \vee \Sigma S_{X} \stackrel{\vartheta_{X}^{-1}}{\longrightarrow} \Sigma_{*}(X) \stackrel{\Sigma_{*}(f)}{\longrightarrow} \Sigma_{*}(Y) \stackrel{\vartheta_{Y}}{\longrightarrow} Y \vee \Sigma S_{Y}
$$

as in 2.1. In other words, for any object $Z$ the induced diagram

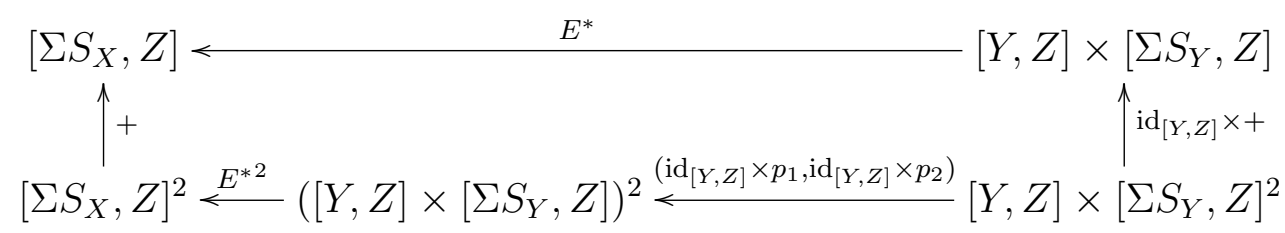

must commute. Now using universal property of suspenders, the entries $[\Sigma ?, Z]$ may be replaced by the isomorphism classes of groupoids $\mathbf{G}_{\text {? }}(Z)$, thus obtaining

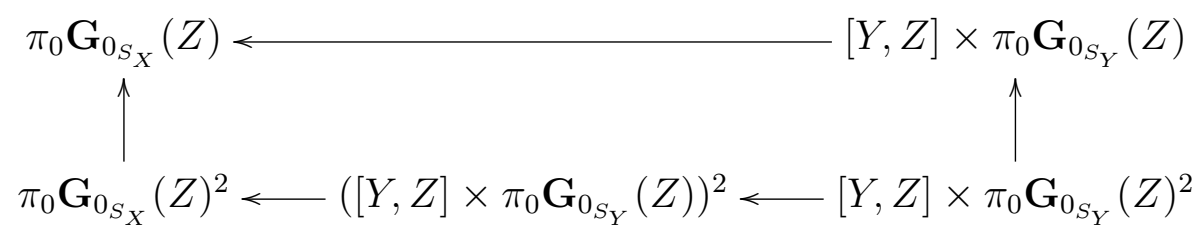

Calculating actions of maps in this diagram in the same way as in [9, 4.3], we see that commutativity of the diagram amounts to conjugatedness of the tracks

$$
\operatorname{id}_{z f} \backslash\left(\operatorname{id}_{z} \cdot \omega_{1}\right) f+\operatorname{id}_{z f} \backslash\left(\operatorname{id}_{z} \cdot \omega_{2}\right) f \text { and } \operatorname{id}_{z f} \backslash\left(\operatorname{id}_{z} \cdot\left(\omega_{1}+\omega_{2}\right)\right) f
$$


in $\operatorname{Aut}\left(!_{Z} 0_{S_{X}}\right)$ for an element $\left(z, \omega_{1}, \omega_{2}\right) \in[Y, Z] \times \mathbf{G}_{0_{S_{Y}}}(Z)^{2}$ given by a map $z: Y \rightarrow Z$ and two tracks in $\operatorname{Aut}\left(!_{Z} 0_{S_{Y}}\right)$, where $\cdot$ and $\backslash$ denote coaction and division maps, as in $[9,4.3]$. But in fact these tracks are easily seen to be equal.

We now turn to the converse statement. Given a linear track category $\mathscr{T}$ with a structure of a $\sum$-theory on $\mathscr{T}$, we know from $[8,4.6]$ that $\mathscr{T}$ has weak sums compatible with the sums in $\mathscr{T}$. Moreover as $\mathscr{T} \simeq$ is a theory of coactions, it is clear that each object of $\mathscr{T}$ has a structure of a weak principal coaction. Thus to have all suspenders it suffices by $[9,4.3]$ to construct suspenders of the maps $0_{S}: S \rightarrow *$, for weak cogroups $S$ in $\mathscr{T}$. For $i_{0}: * \rightarrow \Sigma_{0}$, take the suspension $\Sigma S=E_{*}\left(0_{S}: S \rightarrow *\right)$. Define the universal track

$$
v_{0} \in \operatorname{Aut}\left(S \stackrel{0_{S}}{\longrightarrow} * \stackrel{!_{\Sigma S}}{\longrightarrow} \Sigma S\right)
$$

by $v_{0}=\sigma_{!_{\Sigma S} 0_{S}}(\mathrm{id})$, utilizing the linear track extension action

$$
D_{!_{\Sigma S}}^{\mathscr{T}}=[\Sigma S, \Sigma S] \stackrel{\sigma_{0} S}{\longrightarrow} \operatorname{Aut}\left(!_{\Sigma S} 0_{S}\right)
$$

where the equality follows from 3.1. To show that this indeed has the universal property of a suspender, we have to show the following:

- for any object $Z$ and any track $\eta \in \operatorname{Aut}\left(!_{Z} 0_{S}\right)$ there exists a map $\Sigma_{\eta}$ : $\Sigma S \rightarrow Z$ with $\eta=\Sigma_{\eta} v_{0}$

- for any $h, h^{\prime}: \Sigma S \rightarrow Z$ with $h v_{0}=h^{\prime} v_{0}$ there is a track $\delta: h \rightarrow h^{\prime}$.

Using 3.1, the first condition becomes a tautology, whereas in the second one obtains $h=h^{\prime}$, so trivially one can take for $\delta$ the identity track.

\section{Classification of $\Sigma$-track CAtegories}

An equivalence between theories of coactions $F: \mathbf{T} \rightarrow \mathbf{T}^{\prime}$ is an equivalence of the underlying categories which preserves sums and which carries the coaction structure of an object $X$ in $\mathbf{T}$ to the coaction structure of the object $F(X)$ in $\mathbf{T}^{\prime}$.

5.1. Definition. A weak equivalence between $\Sigma$-track theories is a weak equivalence of the underlying track categories as in $[8,1.5]$ which preserves weak sums and suspenders and for which the induced functor between homotopy categories is an equivalence between theories of coactions.

5.2. Definition. An equivalence $(F, \Phi)$ between $\Sigma$-theories is an equivalence $F: \mathbf{T} \rightarrow \mathbf{T}^{\prime}$ between the underlying theories of coactions which is compatible with the suspension structure, that is for the diagram of functors

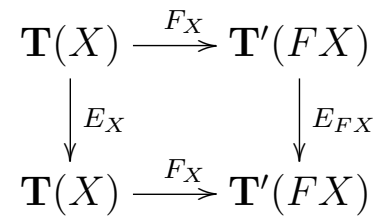

with $F_{X}$ induced by $F$ one has a natural isomorphism $\Phi_{X}: E_{F X} F_{X} \cong F_{X} E_{X}$. 
5.3. Definition. A classifying pair is a pair $(\mathbf{T}, \tau)$ where $\mathbf{T}$ is a $\Sigma$-theory and $\tau$ is an element in the $\Sigma$-cohomology $H_{\Sigma}^{3}(\mathbf{T})$. Two such pairs $(\mathbf{T}, \tau)$ and $\left(\mathbf{T}^{\prime}, \tau^{\prime}\right)$ are equivalent if there exists an equivalence $(F, \Phi)$ with $F: \mathbf{T}^{\prime} \rightarrow \mathbf{T}$ such that $F^{*} \tau=I_{*} \tau^{\prime}$ in $H^{3}\left(\mathbf{T}^{\prime} ; F^{*} D^{\mathbf{T}}\right)$. Here the natural isomorphism $I: D^{\mathbf{T}^{\prime}} \cong F^{*} D^{\mathbf{T}}$ is given by $\Phi$.

Using [8,4.8] and 4.2 we obtain the following classification result.

5.4. Classification. There is a 1-1 correspondence between weak equivalence classes of $\Sigma$-track theories and equivalence classes of classifying pairs.

\section{EXAMPLES}

Since the track category associated to a Quillen model category is both $\Sigma$ representable and $\Omega$-representable (see $[9,1.6]$ ) we get the following result.

6.1. Proposition. Let $\mathbf{Q}_{\mathrm{cf}}$ be the track category of cofibrant and fibrant objects in a Quillen model category and let $\mathscr{T} \subset \mathrm{Q}_{\mathrm{cf}}$ be a full track subcategory. If $\mathscr{T} \simeq$ is a theory of coactions then $\mathscr{T}$ is a $\Sigma$-track theory and if dually $\mathscr{T} \simeq$ is a theory of actions then $\mathscr{T}$ is an $\Omega$-track theory. In particular $\mathscr{T} \simeq$ is a $\Sigma$-theory and a $\Omega$-theory respectively.

A similar result is available for a cofibration category and a fibration category in the sense of Baues [4]. This, in particular, yields the following examples.

6.2. Example. Let $\mathscr{S}(k)$ be the track subcategory of Top* consisting of onepoint unions of spheres $S^{n}$ with $n \geq k$. Then $\mathscr{S}(k)$ is a $\Sigma$-track theory and hence the homotopy category $\mathscr{S}(k) \simeq$ is a $\Sigma$-theory, see 1.1. The partial suspension in $\mathscr{S}(k)_{\simeq}$, in fact, coincides with the partial suspension used in $[5,4,2]$. The partial suspension can be described totally by the ordinary suspension for homotopy groups of spheres and by Whitehead products. The $\Sigma$-track theory $\mathscr{S}(k)$ is classified by an element $\tau \in H_{\Sigma}^{3}\left(\mathscr{S}(k)_{\simeq}\right)$ in the $\Sigma$-cohomology. This is the universal Toda bracket $\langle\mathscr{S}(k)\rangle=\tau$ also considered in [6] and Baues [2].

6.3. Example. Let $\mathscr{K}(k)$ be the track subcategory of Top* consisting of products of Eilenberg-MacLane spaces $K(A, n), n \leq k$, and $A$ a finitely generated abelian group. Then $\mathscr{K}(k)$ is an $\Omega$-track theory and hence the homotopy category $\mathscr{K}(k)_{\simeq}$ is an $\Omega$-theory. The partial loop operation in $\mathscr{K}(k)_{\simeq}$, in fact, coincides with the loop operation $L$ used in $[5,4,2]$. Here $L$ can be described totally by the ordinary loop operation (suspension) in cohomology and by cup products, see [4]. The $\Omega$-track theory $\mathscr{K}(k)$ is classified by an element $\tau \in H_{\Omega}^{3}\left(\mathscr{K}(k)_{\simeq}\right)$ in the $\Omega$-cohomology. This is the universal Toda bracket $\langle\mathscr{K}(k)\rangle=\tau$ also considered by Baues and Dreckmann [6] and Baues [2].

Recall that an additive category $\mathbf{A}$ is a category enriched in abelian groups which has sums. These sums are then also products (termed direct sums) denoted $A \oplus B$; see [17]. Each object is canonically an abelian group object by 
$(1,1): A \oplus A \rightarrow A$ and all maps in $\mathbf{A}$ are linear. A functor between additive categories is additive if it preserves biproducts.

6.4. Lemma. An additive category A together with an additive functor $\sigma$ : $\mathbf{A} \rightarrow \mathbf{A}$ has canonically the structure of both a $\Sigma$-theory and a $\Omega$-theory.

Proof. We simply define the suspender in 2.4 by $\Sigma_{*}(X)=X \oplus \sigma(X)$. Dually we define a looper by $\Omega_{*}(X)=X \oplus \sigma(X)$. One readily checks the properties in 2.4 and the dual properties respectively.

The $\Sigma$-cohomology of the $\Sigma$-theory $(\mathbf{A}, \sigma)$ in 6.4 is the cohomology

$$
H_{\Sigma}^{n}(\mathbf{A}, \sigma)=H^{n}(\mathbf{A} ; \operatorname{Hom}(\sigma,-)),
$$

where $\operatorname{Hom}(\sigma,-)$ is the bifunctor $\mathbf{A}^{\text {op }} \times \mathbf{A} \rightarrow \mathbf{A b}$ which carries $(X, Y)$ to $\operatorname{Hom}(\sigma X, Y)$. Moreover the $\Omega$-cohomology of the $\Omega$-theory $(\mathbf{A}, \sigma)$ is the cohomology

$$
H_{\Omega}^{n}(\mathbf{A}, \sigma)=H^{n}(\mathbf{A} ; \operatorname{Hom}(-, \sigma)),
$$

where the bifunctor $\operatorname{Hom}(-, \sigma)$ carries $(X, Y)$ to $\operatorname{Hom}(X, \sigma Y)$. Here Hom denotes the set of morphisms in $\mathbf{A}$ which is an abelian groups since $\mathbf{A}$ is an additive category. The cohomology groups above are special cases of those by Baues and Wirsching [2] which are in particular studied by Jibladze and Pirashvili [15].

Let $R$ be a commutative ring with unit and let $\operatorname{Mod}_{*}(R)$ be the category of $\mathbb{Z}$-graded $R$-modules $X=\left(X_{i}\right)_{i \in \mathbb{Z}}$ with the graded tensor product $X \otimes Y$ given by

$$
(X \otimes Y)_{n}=\bigoplus_{i+j=n} X_{i} \otimes_{R} Y_{j}
$$

Here $X$ is non-negatively graded if $X_{i}=0$ for $i<0$. A non-negatively graded $R$-module $A$ which has the structure of a monoid in $\left.\operatorname{Mod}_{*}(R), \otimes\right)$ is termed a graded algebra. Let $R$ be the graded $R$-module concentrated in degree 0 and given by the ring $R$. Then of course $R$ is also a graded algebra; in fact, the initial object in the category of graded algebras. A map $\varepsilon: A \rightarrow R$ between graded algebras is termed an augmentation of $A$.

6.5. Example. Let $A$ be a graded algebra and let $\mathbf{T}_{A}$ be the category of $\mathbb{Z}$ graded finitely generated free left $A$-modules. Objects are direct sums of objects $A[n]$ with $n \geq 0$, where $A[n]$ is the free left $A$-module generated by a single element $[n]$ in degree $n$. The elements of $A[n]$ are of the form $a \cdot[n]$ with $a \in A$ and degree $|a \cdot[n]|=|a|+n$. Morphisms $f: A[n] \rightarrow A[m]$ are determined by the element $\alpha \in A$ with $f[n]=\alpha \cdot[m]$ and we write $f=\alpha^{*}$ if $f(a \cdot[n])=a \cdot \alpha \cdot[m]$. We define the shift functor

$$
\sigma: \mathbf{T}_{A} \rightarrow \mathbf{T}_{A}
$$

to be the unique additive functor which carries $A[n]$ to $A[n+1]$ and carries $f=\alpha^{*}: A[n] \rightarrow A[m]$ to $\alpha^{*}: A[n+1] \rightarrow A[m+1]$. Hence by 6.4 the category $\mathbf{T}_{A}$ together with $\sigma$ is canonically both a $\Sigma$-theory and a $\Omega$-theory. 


\section{ACKNOWLEDGEMENT}

The second author gratefully acknowledges hospitality of the Max Planck Institut für Mathematik, Bonn and of the Université Catholique de Louvain, Louvain-la-Neuve.

\section{REFERENCES}

1. J. F. Adams, On the non-existence of elements of Hopf invariant one. Ann. Math. 72(1960) 10-104.

2. H.-J. BAUES, On the cohomology of categories, universal Toda brackets and homotopy pairs. K-theory 11(1997), 259-285.

3. H.-J. BAues, Combinatorial homotopy and 4-dimensional complexes. De Gruyter Expositions in Mathematics, 2. Walter de Gruyter, Berlin etc., 1991

4. H.-J. Baues, Algebraic homotopy. Cambridge studies in advanced mathematics 15, Cambridge University Press, Cambridge, 1989.

5. H.-J. Baues, Obstruction theory. Lecture Notes in Math. 628, Springer, 1974.

6. H.-J. Baues and W. Dreckmann, The cohomology of homotopy categories and the general linear group. K-theory $\mathbf{3}(1989)$, No. 4, 307-338.

7. H.-J. Baues and P. Goerss, A homotopy spectral sequence for the computation of homotopy groups. Topology 39(2000), 161-192.

8. H.-J. Baues and M. Jibladze, Classification of abelian track categories. Preprint.

9. H.-J. Baues and M. Jibladze, Suspension and loop objects and representability of tracks. Georgian Math. J. 8(2001), No. 4, 683-696.

10. H.-J. Baues and M. Jibladze, The Steenrod algebra and theories associated to Hopf algebras. Preprint.

11. H.-J. Baues and G. Wirsching, Cohomology of small categories. J. Pure Appl. Algebra 38(1985), 187-211.

12. D. Blanc, Higher homotopy operations and the realizability of homotopy groups. Proc. London Math. Soc. 70(1995), 214-240.

13. W. G. Dwyer, D. M. Kan and C. R. Stower, The bigraded homotopy groups $\pi_{i, j} X$ of a pointed simplicial space X. J. Pure Appl. Algebra 103(1995), 167-188.

14. B. Gray, Homotopy theory. Academic Press, N.Y. etc., 1975.

15. M. Jibladze and T. Pirashvili, Cohomology of algebraic theories. J. Algebra 137(1991), No. 2, 253-296.

16. F. W. Lawvere, Functorial semantics of algebraic theories. Proc. Nat. Acad. Sci. USA 50(1963), 869-873.

17. S. Mac Lane, Categories for the working mathematician. Graduate Texts in Math., Springer, 1971.

18. C. R. Stover, A van Kampen spectral sequence for higher homotopy groups. Topology 29(1990), 9-26.

(Received 28.11.2000)

Authors' addresses:

H.-J. Baues

Max-Planck-Institut fuer Mathematik 
Vivatsgasse 7, D-53111 Bonn

Germany

E-mail: baues@mpim-bonn.mpg.de

M. Jibladze

A. Razmadze Mathematical Institute Georgian Academy of Sciences

1, M. Aleksidze St., Tbilisi 380093

Georgia

E-mail: jib@rmi.acnet.ge 